\title{
Was ist neu bei Grundwasser? - Staffelstab übernommen
}

\author{
Johannes Barth • Rudolf Liedl
}

Eingang des Beitrages: 13. 12.2009 / Eingang des überarbeiteten Beitrages: 14. 12.2009 / Online veröffentlicht: 28. 1.2010

(C) Springer-Verlag 2010

\section{Liebe Leserinnen und Leser,}

mit seiner Abhandlung zum Prinzip der kommunizierenden Röhren legte Blaise Pascal 1648 auch wichtige Grundlagen für die Hydrogeologie. Sehr wahrscheinlich hatte er aber kaum eine Vorstellung davon, wie dieses Prinzip viele Jahre später als Einleitung einer Ausgabe von Grundwasser verwendet werden könnte, geht es uns im Folgenden doch eher um die Kommunikation im übertragenen Sinne, die - ähnlich wie bei kommunizierenden Röhren - bei Grundwasser exzellent funktioniert.

Vergangenen November fand in Erlangen ein Treffen statt, bei dem Mario Schirmer, Ruth Kaufmann-Knoke und Jörg Böger die Übergabe der Editorenschaft von Grundwasser an uns einleiteten. Bereits nach wenigen Minuten war klar, dass wir Verantwortung für ein sehr gut organisiertes Kommunikations- und Sprachrohr übernehmen, welches die Belange einer wachsenden Fachgruppe und Interessengemeinschaft in der Hydrogeologie und damit verbundener Themen abdeckt. Dafür gilt unser Dank den Genannten sowie allen, die durch aktives Mitwirken, Beiträge, Kommentare und sonstige Aktivitäten in der FH-DGG Grundwasser zu der Zeitschrift gemacht haben, die sie jetzt ist. Aufgrund der hervorragenden Ausgangslage sind wir sehr optimistisch, dass Grundwasser als Fachzeitschrift auch weiterhin von der exzellenten Kommunikation in der FHDGG getragen wird, aber auch ihrerseits dazu beiträgt.

Prof. Dr. J. Barth $(\bowtie)$

GeoZentrum Nordbayern, Lehrstuhl für Angewandte Geologie, Friedrich-Alexander-Universität Erlangen-Nürnberg,

Schlossgarten 5, 91054 Erlangen, Deutschland

E-Mail: barth@geol.uni-erlangen.de

Prof. Dr. R. Liedl

Inst. f. Grundwasserwirtschaft, TU Dresden, 01062 Dresden, Deutschland

E-Mail: rudolf.liedl@mailbox.tu-dresden.de
„Was ist neu?“ mögen Sie sich anlässlich des Editorenwechsels fragen und zunächst können wir drei Dinge nennen:

- Die Zeitschrift wird nunmehr von einem gleichberechtigten Team von zwei Editoren geleitet - eine Entwicklung, die am besten der wachsenden Diversität und Anzahl der Beiträge gerecht wird.

- Bereits seit dem Vorjahr beinhaltet Grundwasser neben Nachrichten und Fachbeiträgen auch Kurzbeiträge. Diese sind ein Instrument, um der Gemeinschaft der Hydrogeologen neue Methoden, technische Entwicklungen, Modelle oder Projekte auf knappe und informative Weise nahe zu bringen.

- Die Gruppe der Assoziierten Editoren wurde neu zusammengesetzt und erweitert. Hierbei ging es darum, Spezialistinnen und Spezialisten aus verschiedenen Ländern, mit unterschiedlichen Expertisen und diversen Arbeitsumfeldern zu finden. Das neue Team der Assoziierten Editoren umfasst Mitglieder von Behörden, Forschungseinrichtungen, Universitäten und Beratungsunternehmen aus Deutschland, Österreich, Saudi Arabien, der Schweiz, Frankreich, Kanada, Großbritannien und Australien.

Alle diese Punkte zeigen, dass Hydrogeologie ein wachsendes und lebendiges Fach in Wissenschaft und Gesellschaft ist, welches interdisziplinäre Zusammenarbeit sucht und umsetzt. Natürlich kann dies viel besser in Teamarbeit geschehen. Damit verbunden unser Aufruf an Sie: Tragen Sie gerne etwas bei! Verfassen Sie Beiträge und teilen Sie mit uns Neuigkeiten der Hydrogeologie im Nachrichtenteil! Wir freuen uns auf eine aktive Kommunikation.

Ihr

Johannes Barth \& Rudolf Liedl

N.B.: Wir starten die Übergabe mit einem Themenheft zu Hydrogeologie in Österreich und haben zu diesem besonderen Anlass ein zweites Editorial zum Thema von Steffen Birk und Thilo Hofmann. 